Qing Shi, Qi-Long Zhang*, Hong Xu and Ya-Li Huang

\title{
Crystal structure of $3,3^{\prime}, 3^{\prime \prime}-\left(\left(1 E, 1^{\prime} E, 1^{\prime \prime} E\right)\right.$ - ((nitrilotris(ethane-2,1-diyl))tris(azaneylylidene)) tris(methaneylylidene))tris(4-hydroxy- 1-naphthaldehyde) monohydrate, $\mathrm{C}_{42} \mathrm{H}_{36} \mathrm{~N}_{4} \mathrm{O}_{6} \cdot \mathrm{H}_{2} \mathrm{O}$
}

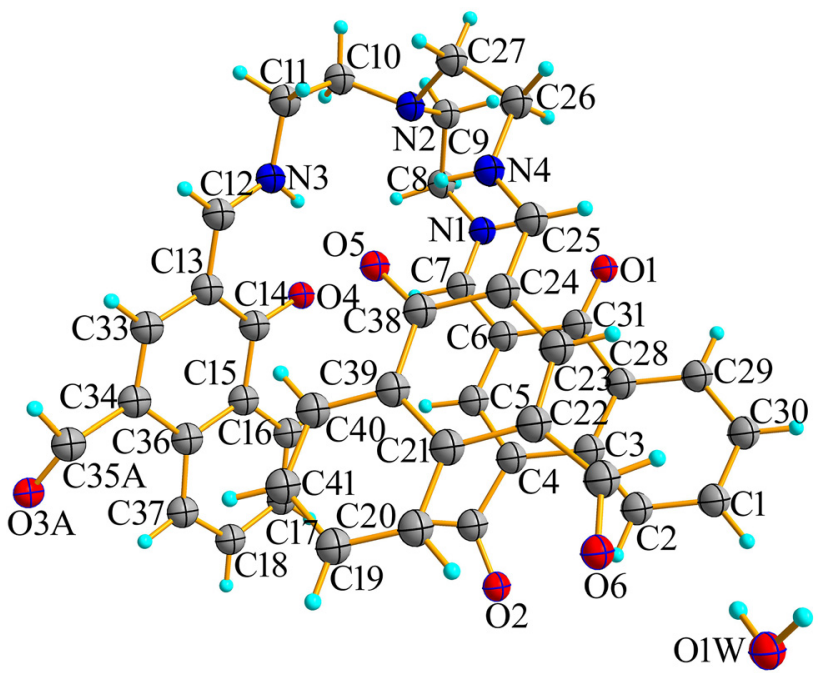

https://doi.org/10.1515/ncrs-2021-0120

Received March 31, 2021; accepted April 12, 2021;

published online April 26, 2021

Table 1: Data collection and handling.

\begin{tabular}{ll}
\hline Crystal: & Yellow block \\
Size: & $0.24 \times 0.23 \times 0.21 \mathrm{~mm}$ \\
Wavelength: & Mo $K \alpha$ radiation $(0.71073 \AA)$ \\
$\mu:$ & $0.09 \mathrm{~mm}^{-1}$ \\
Diffractometer, scan mode: & BRUKER APEX-II, $\varphi$ and $\omega$ \\
$\theta_{\text {max }}$, completeness: & $26.0^{\circ}, 99 \%$ \\
$N(h k l)_{\text {measured }}, N(h k l)_{\text {unique }}, R_{\text {int }}:$ & $47,868,6780,0.040$ \\
$C$ riterion for $I_{\text {obs }}, N(h k l)_{\text {gt }}:$ & $I_{\text {obs }}>2 \sigma\left(I_{\text {obs }}\right), 5360$ \\
$N(\text { param })_{\text {refined }}:$ & 495 \\
Programs: & Olex2 [1], Bruker [2], \\
& Shelx [3], Diamond [4] \\
\hline
\end{tabular}

The molecular structure is shown in the Figure. Table 1 contains crystallographic data and Table 2 contains the list of the atoms including atomic coordinates and displacement parameters.

\section{Source of material}

To a solution of 1-naphthol (1 g, $6.9 \mathrm{mmol})$ in trifluoroacetic acid $(10 \mathrm{~mL})$ was added hexamethylenetetramine $(1.94 \mathrm{~g}$, $13.8 \mathrm{mmol})$. The above mixture was heated and stirred for about $1 \mathrm{~h}$. Then concentrated sulfuric acid with a concentration of $33 \%(10 \mathrm{~mL})$ was added to the stirred solution. The reaction mixture was then stirred and heated for $1 \mathrm{~h}$. Then water was added and the whole solution was extracted by ethyl acetate $(30 \mathrm{~mL} \times 2)$. The mixture was washed with brine and dried and filtered by adding anhydrous magnesium sulfate. The reaction mixture is further purified by column chromatography on silica gel, using ethyl acetate: $n$-hexane (3:7) as the eluant to afford 1-hydroxy-2,4-diformylnaphthalene as yellow oil. To the solution, 1-hydroxy-2,4-diformylnaphthalene $(0.60 \mathrm{~g}$, $3 \mathrm{mmol}$ ) in $50 \mathrm{~mL}$ ethanol was added tri (2-aminoethyl)

*Corresponding author: Qi-Long Zhang, School of Basic Medical Science, Guizhou Medical University, Guiyang, 550025, People's 0000-0002-6715-9548

Qing Shi, School of Public Health, The Key Laboratory of Environmental Pollution Monitoring and Disease Control, Ministry of Education, Guizhou Medical University, Guiyang, 550025, People’s Republic of China

Hong Xu and Ya-Li Huang, School of Basic Medical Science, Guizhou Medical University, Guiyang, 550025, People’s Republic of China 
Table 2: Fractional atomic coordinates and isotropic or equivalent isotropic displacement parameters $\left(\AA^{2}\right)$.

\begin{tabular}{|c|c|c|c|c|}
\hline Atom & $x$ & $y$ & $z$ & $U_{\text {iso }} * / U_{\text {eq }}$ \\
\hline 01 & $0.67282(11)$ & $0.17824(11)$ & $-0.05327(10)$ & 0.0549 (3) \\
\hline 02 & $0.6140(2)$ & $-0.15380(18)$ & 0.20935 (17) & $0.1136(7)$ \\
\hline 04 & $0.56490(10)$ & $0.41825(11)$ & $0.38665(9)$ & $0.0540(3)$ \\
\hline 05 & $0.85768(14)$ & 0.40747 (13) & $0.36701(11)$ & $0.0694(4)$ \\
\hline 06 & 1.01414 (17) & $-0.07558(14)$ & $0.23454(16)$ & $0.0928(5)$ \\
\hline N1 & $0.59053(11)$ & $0.34105(11)$ & $0.08463(11)$ & $0.0437(3)$ \\
\hline $\mathrm{H} 1$ & 0.6117 & 0.3148 & 0.0225 & $0.052^{\star}$ \\
\hline $\mathrm{N} 2$ & $0.76003(11)$ & $0.59836(11)$ & $0.22108(10)$ & $0.0415(3)$ \\
\hline N3 & $0.74550(12)$ & $0.61887(12)$ & $0.45091(11)$ & $0.0451(3)$ \\
\hline H3 & 0.6848 & 0.5629 & 0.3989 & $0.054^{*}$ \\
\hline N4 & $0.90252(13)$ & $0.43894(13)$ & $0.19194(12)$ & $0.0516(3)$ \\
\hline $\mathrm{H} 4$ & 0.8802 & 0.4655 & 0.2534 & $0.062^{\star}$ \\
\hline $\mathrm{C} 1$ & $0.73993(17)$ & $-0.20695(16)$ & $-0.08058(17)$ & $0.0616(5)$ \\
\hline $\mathrm{H} 1 \mathrm{~A}$ & 0.7592 & -0.2785 & -0.1001 & $0.074^{*}$ \\
\hline $\mathrm{C} 2$ & 0.70537 (15) & $-0.15823(15)$ & $0.01654(16)$ & $0.0526(4)$ \\
\hline $\mathrm{H} 2$ & 0.7016 & -0.1971 & 0.0626 & $0.063^{*}$ \\
\hline $\mathrm{C} 3$ & $0.67525(13)$ & $-0.04999(13)$ & 0.04830 (13) & $0.0402(3)$ \\
\hline $\mathrm{C} 4$ & $0.63472(14)$ & $0.00249(15)$ & $0.14884(13)$ & $0.0446(4)$ \\
\hline $\mathrm{C} 5$ & 0.61104 (14) & $0.10929(14)$ & $0.17492(13)$ & $0.0431(3)$ \\
\hline $\mathrm{H} 5$ & 0.5863 & 0.1427 & 0.2404 & $0.052^{\star}$ \\
\hline $\mathrm{C} 6$ & $0.62166(12)$ & $0.17220(13)$ & $0.10878(12)$ & $0.0375(3)$ \\
\hline $\mathrm{C} 7$ & $0.59061(13)$ & 0.27939 (13) & $0.14202(12)$ & $0.0406(3)$ \\
\hline $\mathrm{H} 7$ & 0.5686 & 0.3081 & 0.2096 & $0.049^{\star}$ \\
\hline $\mathrm{C} 8$ & 0.55727 (15) & 0.45134 (15) & 0.11765 (15) & 0.0504 (4) \\
\hline $\mathrm{H} 8 \mathrm{~A}$ & 0.5375 & 0.4724 & 0.1896 & $0.060^{*}$ \\
\hline $\mathrm{H} 8 \mathrm{~B}$ & 0.4867 & 0.4367 & 0.0633 & $0.060^{*}$ \\
\hline $\mathrm{C} 9$ & $0.65926(15)$ & $0.55664(14)$ & $0.12552(14)$ & 0.0498 (4) \\
\hline $\mathrm{H} 9 \mathrm{~A}$ & 0.6868 & 0.5310 & 0.0572 & $0.060^{*}$ \\
\hline $\mathrm{H} 9 \mathrm{~B}$ & 0.6301 & 0.6240 & 0.1326 & $0.060^{*}$ \\
\hline $\mathrm{C} 10$ & $0.75461(17)$ & 0.70437 (14) & 0.31734 (14) & $0.0506(4)$ \\
\hline $\mathrm{H} 10 \mathrm{~A}$ & 0.6712 & 0.6981 & 0.3161 & $0.061^{*}$ \\
\hline $\mathrm{H} 10 \mathrm{~B}$ & 0.7928 & 0.7781 & 0.3110 & $0.061^{*}$ \\
\hline C11 & $0.81524(17)$ & 0.71464 (15) & $0.42733(14)$ & $0.0531(4)$ \\
\hline $\mathrm{H} 11 \mathrm{~A}$ & 0.8948 & 0.7082 & 0.4247 & $0.064^{*}$ \\
\hline $\mathrm{H} 11 \mathrm{~B}$ & 0.8241 & 0.7939 & 0.4869 & $0.064^{*}$ \\
\hline C12 & $0.76748(14)$ & $0.61120(14)$ & 0.54377 (13) & $0.0440(4)$ \\
\hline $\mathrm{H} 12$ & 0.8319 & 0.6725 & 0.6009 & $0.053^{*}$ \\
\hline C13 & 0.70084 (14) & 0.51669 (14) & $0.56429(12)$ & $0.0411(3)$ \\
\hline C14 & $0.60062(13)$ & 0.41909 (14) & $0.47886(12)$ & $0.0419(3)$ \\
\hline C15 & $0.54290(14)$ & 0.31796 (14) & 0.50257 (13) & $0.0445(4)$ \\
\hline $\mathrm{C} 16$ & $0.45017(16)$ & 0.21765 (17) & 0.41887 (15) & $0.0571(4)$ \\
\hline H16 & 0.4248 & 0.2179 & 0.3509 & $0.069^{\star}$ \\
\hline C17 & $0.39626(19)$ & 0.11893 (19) & $0.43580(18)$ & $0.0708(6)$ \\
\hline $\mathrm{H} 17$ & 0.3345 & 0.0527 & 0.3798 & $0.085^{*}$ \\
\hline C18 & $0.4344(2)$ & 0.11853 (19) & $0.53676(18)$ & $0.0728(6)$ \\
\hline H18 & 0.3981 & 0.0512 & 0.5479 & $0.087^{*}$ \\
\hline C19 & $0.87199(16)$ & 0.10065 (18) & 0.50848 (18) & $0.0615(5)$ \\
\hline H19 & 0.8667 & 0.0538 & 0.5474 & $0.074^{*}$ \\
\hline $\mathrm{C} 20$ & 0.91004 (15) & $0.06424(15)$ & 0.41139 (17) & $0.0543(4)$ \\
\hline $\mathrm{H} 20$ & 0.9301 & -0.0071 & 0.3854 & $0.065^{\star}$ \\
\hline $\mathrm{C} 21$ & 0.91929 (13) & 0.13297 (14) & $0.35022(14)$ & 0.0437 (4) \\
\hline $\mathrm{C} 22$ & $0.95756(14)$ & 0.09777 (14) & 0.24645 (14) & $0.0467(4)$ \\
\hline $\mathrm{C} 23$ & 0.95906 (14) & $0.16782(14)$ & 0.19058 (14) & $0.0466(4)$ \\
\hline $\mathrm{H} 23$ & 0.9816 & 0.1425 & 0.1231 & $0.056^{*}$ \\
\hline C24 & 3) & 4) & 3) & 0432 \\
\hline
\end{tabular}

Table 2: (continued)

\begin{tabular}{|c|c|c|c|c|}
\hline Atom & $x$ & $y$ & $z$ & $U_{\text {iso }}{ }^{*} / U_{\text {eq }}$ \\
\hline $\mathrm{C} 25$ & $0.93273(14)$ & $0.34164(15)$ & $0.16545(14)$ & 0.0477 (4) \\
\hline 25 & 0.9589 & 0.3132 & 0.1001 & $0.057^{*}$ \\
\hline C26 & 0.90287 (18) & $0.50816(18)$ & $0.12679(16)$ & $0.0585(5)$ \\
\hline $\mathrm{H} 26 \mathrm{~A}$ & 0.8428 & 0.4589 & 0.0564 & $0.070^{*}$ \\
\hline $\mathrm{H} 26 \mathrm{~B}$ & 0.9808 & 0.5284 & 0.1105 & $0.070^{*}$ \\
\hline C27 & $0.87601(16)$ & $0.62363(16)$ & 0.19195 (15) & 0.0527 (4) \\
\hline $\mathrm{H} 27 \mathrm{~A}$ & 0.9392 & 0.6747 & 0.2600 & $0.063^{*}$ \\
\hline H27B & 0.8753 & 0.6684 & 0.1478 & $0.063^{*}$ \\
\hline 28 & $0.68638(13)$ & 0.00916 (13) & $-0.02121(12)$ & $0.0394(3)$ \\
\hline C29 & $0.72153(16)$ & $-0.04322(15)$ & $-0.12058(14)$ & $0.0523(4)$ \\
\hline $\mathrm{H} 29$ & 0.7280 & -0.0048 & -0.1670 & $0.063^{*}$ \\
\hline 30 & $0.74651(18)$ & $-0.15052(17)$ & $-0.15024(16)$ & $0.0632(5)$ \\
\hline $\mathrm{H} 30$ & 0.7679 & -0.1855 & -0.2173 & $0.076^{*}$ \\
\hline 31 & $0.66041(13)$ & 0.12407 (13) & $0.00769(12)$ & $0.0391(3)$ \\
\hline C32 & $0.6099(2)$ & $-0.0547(2)$ & 0.22167 (19) & $0.0730(6)$ \\
\hline H32 & 0.5881 & -0.0097 & 0.2857 & $0.088^{*}$ \\
\hline 33 & $0.73386(15)$ & 0.51555 (15) & $0.66757(12)$ & $0.0454(4)$ \\
\hline H33 & 0.7973 & 0.5810 & 0.7223 & $0.054^{\star}$ \\
\hline 34 & $0.67810(15)$ & $0.42366(15)$ & $0.69214(13)$ & $0.0469(4)$ \\
\hline C36 & $0.58160(15)$ & $0.31893(15)$ & $0.60675(13)$ & $0.0468(4)$ \\
\hline 37 & $0.52430(18)$ & $0.21533(18)$ & $0.62054(17)$ & $0.0626(5)$ \\
\hline H37 & 0.5481 & 0.2128 & 0.6877 & $0.075^{\star}$ \\
\hline C38 & $0.88971(15)$ & 0.31522 (15) & $0.33144(14)$ & $0.0471(4)$ \\
\hline C39 & $0.88716(14)$ & 0.23985 (15) & $0.39186(14)$ & $0.0450(4)$ \\
\hline $\mathrm{C} 40$ & 0.84899 (17) & $0.27474(18)$ & $0.49113(16)$ & $0.0580(4)$ \\
\hline $\mathrm{H} 40$ & 0.8283 & 0.3457 & 0.5183 & $0.070^{*}$ \\
\hline C41 & $0.84143(17)$ & 0.20636 (19) & $0.54918(18)$ & $0.0632(5)$ \\
\hline H41 & 0.8160 & & 52 & $0.076^{*}$ \\
\hline C42 & $0.99614(17)$ & $-0.00763(16)$ & $0.19591(18)$ & $0.0621(5)$ \\
\hline $\mathrm{H} 42$ & 1.0087 & -0.0255 & 0.1248 & $0.074^{\star}$ \\
\hline $03^{\mathrm{a}}$ & $0.7964(2)$ & $0.5211(2)$ & 0.87907 (15) & $0.0734(8)$ \\
\hline $\mathrm{C} 35 \mathrm{~A}^{\mathrm{b}}$ & $0.724(3)$ & $0.425(2)$ & $0.800(2)$ & $0.0602(18)$ \\
\hline $\mathrm{H} 35 \mathrm{~A}^{\mathrm{b}}$ & 0.6984 & 0.3542 & 0.8096 & $0.072^{*}$ \\
\hline $03 A^{b}$ & $0.6662(6)$ & $0.3918(5)$ & $0.8569(4)$ & $0.096(2)$ \\
\hline $\mathrm{C} 35^{\mathrm{a}}$ & $0.7162(12)$ & $0.4418(9)$ & 0.8064 (11) & $0.0602(18)$ \\
\hline $\mathrm{H} 35^{\mathrm{a}}$ & 0.7905 & 0.5011 & 0.8467 & $0.072^{\star}$ \\
\hline $01 W^{c}$ & $1.1141(3)$ & $-0.2607(3)$ & $0.1164(3)$ & $0.0865(7)$ \\
\hline$H 1 W A^{c}$ & 1.1767 & -0.2286 & 0.1001 & $0.130^{\star}$ \\
\hline$H_{1 W B^{c}}$ & 1.0670 & -0.2215 & 0.1104 & $0.130^{*}$ \\
\hline $01 X^{d}$ & $1.1651(4)$ & $-0.2285(4)$ & $0.1835(4)$ & $0.0865(7)$ \\
\hline$H 1 X A^{d}$ & 1.1426 & -0.1661 & 0.2104 & $0.130 *$ \\
\hline$H 1 X^{d}$ & 1.2338 & -0.2080 & 0.1707 & $0.130^{*}$ \\
\hline
\end{tabular}

${ }^{a}$ Occupancy: 0.672(4), boccupancy: 0.328(4), 'occupancy: 0.561(4), doccupancy: 0.439(4).

amine ( $0.15 \mathrm{~g}, 1 \mathrm{mmol})$. The reaction mixture was refluxed for $8 \mathrm{~h}$, then the solution was cooled to room temperature. The yellow precipitated residue was removed from the solution by filtration and then washed with ethanol and trichloromethane three times. Then a share of the product ( $0.15 \mathrm{mmol})$ was dissolved in $20 \mathrm{~mL}$ DMF solution. Single crystals were obtained from DMF by slow evaporation at room temperature. 


\section{Experimental details}

Using Olex2 [1], the structure was solved using Charge Flipping and refined with the ShelXL [3] refinement. All hydrogen atoms were positioned geometrically, with the $d(\mathrm{C}-\mathrm{H})=0.97-0.99 \AA, U_{\text {iso }}(\mathrm{H})=1.2$ times $U_{e q}(\mathrm{C})$ and $U_{\text {iso }}(\mathrm{H})=1.5$ times $U_{\text {eq }}(\mathrm{O})$.

\section{Comment}

Naphthaldehyde based nitrogen rich Schiff bases bear excellent coordinating abilities to act as either multidentate ligands or bridging blocks in supramolecular chemistry and have been highlighted in the literature due to novel structural architectures as well as potential applications of their complexes [5]. In the crystal structure of the title compound, the asymmetric unit of the title compound consists of one formula unit of the target molecule and one water molecule. The bond lengths and bond angles are in the normal ranges [6]. The crystal structure shows that the hydrogen on the hydroxyl oxygen atom of phenol moiety is transferred to the nitrogen atom of imine to form keto-enamine [6]. There are intramolecular hydrogen bonds (for example: $\mathrm{N} 1-\mathrm{H} 1 \cdots \mathrm{O} 1$, $\mathrm{N} 3-\mathrm{H} 3 \cdots \mathrm{O} 4, \mathrm{~N} 4-\mathrm{H} 4 \cdots \mathrm{O} 5, \mathrm{C} 7-\mathrm{H} 7 \cdots \mathrm{O} 4)$ in the molecules. Through the hydrogen bond of O1W-H1WA $\cdots 01$, O1W-H1WB $\cdots 06$ the interaction between the target and water molecules extends into a dimer structure. The water molecule and one of the aldehyde groups show a disorder.

Author contributions: All the authors have accepted responsibility for the entire content of this submitted manuscript and approved submission.

Research funding: This work was supported by and the National Natural Science Foundation of China (22065009, 22066007) and Preventive Medicine (No. 2017[85]), Science and Technology Foundation of Guizhou Province (grant numbers [2019]2792, [2018]5779-14).

Conflict of interest statement: The authors declare no conflicts of interest regarding this article.

\section{References}

1. Dolomanov O. V., Bourhis L. J., Gildea R. J., Howard J. A. K., Puschmann H. OLEX2: a complete structure solution, refinement and analysis program. J. Appl. Crystallogr. 2009, 42, 339-341.

2. Bruker. APEX2, SAINT and SADABS; Bruker AXS Inc.: Madison, Wisconsin, USA, 2012.

3. Sheldrick G. M. Crystal structure refinement with SHELXL. Acta Crystallogr. 2015, C71, 3-8.

4. Brandenburg K. DIAMOND. Visual Crystal Structure Information System. Ver. 4.0; Crystal Impact: Bonn, Germany, 2015.

5. Naskar B., Modak R., Mait D. K., Drew M. G. B., Bauza A., Frontera A., Mukhopadhyay C. D., Mishra S., Saha K. D., Goswami S. A Schiff base platform: structures, sensing of $\mathrm{Zn}$ (II) and PPi in aqueous medium and anticancer activity. Dalton Trans. 2017, 46, 9498-9510.

6. Park H., Kim J., Kwon E., Kim T. H. Crystal structure of flucetosulfuron. Acta Crystallogr. 2017, E73, 1439-1442. 